\title{
EL ROL DE LA ARQUITECTURA EN EL BICENTENARIO
}

\author{
THE ROLE OF ARCHITECTURE IN THE BICENTENNIAL \\ Mayda Alvina Nieva Villegas ${ }^{1 *}$, Crhistian Edinson Murga Tirado ${ }^{1}$ \\ maydanieva@unat.edu.pe ; crhistianmurga@unat.edu.pe \\ 'Universidad Nacional Autónoma de Tayacaja, Huancavelica, Perú \\ *Correspondencia: Mayda Alvina Nieva Villegas. Email: maydanieva@unat.edu.pe
}

Recibido: 17.05.21 | Aprobado: 25.06.21

\section{RESUMEN}

Esta propuesta reflexiva está dirigida a los ciudadanos consientes que quieren que la arquitectura cumpla un rol de liderazgo en los cambios sustantivos en la organización del espacio urbano en la planificación de las ciudades a raíz que se acerca vertiginosamente al bicentenario de Perú (Velaochaga-Sacio, 2020) El diseño de la investigación es de tipo mixto, descriptivo-comparativo, debido a la degradación, deterioro, destrucción y alteración que vienen sufriendo las ciudades; y tiene un enfoque cualitativo.

Las ciudades en los momentos actuales vienen atravesando una crisis y degradación de su patrimonio arquitectónico monumental, a esto se suma el mal manejo de las administraciones ediles en cuanto al desarrollo urbano y mal uso de las reglamentaciones conspirando con la esencia del pensamiento contemporáneo en su producción creativa para el desarrollo de las ciudades. Los ciudadanos no sienten que existan cambios sustanciales para una mejor convivencia. En 1921 en el Centenario el slogan fue "hombre nuevo" en el sesquicentenario 1971 "el poder popular", en los momentos actuales se le denomina "Año del Bicentenario del Perú: 200 años de Independencia" pero no se nota cambio alguno donde se sienta la independencia. Las personas necesitan un cambio de la arquitectura a raíz de los momentos pandémicos y que los objetos arquitectónicos deban brindar satisfacciones físicas, psicológicas, espirituales para que los ciudadanos puedan cumplir la actividad principal de la habitabilidad y la socialización en su máxima dimensión de confortabilidad dado a que los espacios están fragmentados y no cumplen con su función. Palabras clave: Rol, reflexiva, degradación, producción creativa, bicentenario.

\begin{abstract}
This reflective proposal is aimed at conscious citizens that we want architecture to play a leading role in the substantive changes in the organization of urban space in city planning as we are rapidly approaching the bicentennial of our country. The research design is of a mixed, descriptive-comparative type, due to the degradation, deterioration, destruction and alteration that our cities are suffering; and it has a qualitative approach.

The cities at the present time are going through a crisis and degradation of their monumental architectural heritage, to this is added the mismanagement of municipal administrations in terms of urban development and misuse of regulations conspiring with the essence of contemporary thought in their production creative for the development of cities. Citizens do not feel that there are substantial changes for a better coexistence. In 1921 in the Centennial the slogan was "new man" in the 1971 sesquicentennial "the popular power", at the present time it is called "Year of the Bicentennial of Peru: 200 years of Independence" but no change is noticed that is felt the independence. People need a change in architecture as a result of pandemic moments and that architectural object must provide physical, psychological, spiritual satisfaction so that citizens can fulfill the main activity of habitability and socialization in its maximum dimension of comfort given to that spaces are fragmented and do not fulfill their function.
\end{abstract}

Keywords: Role, reflective, degradation, creative production, bicentennial. 


\section{INTRODUCCIÓN}

El bicentenario de nuestra independencia se da en una realidad con grave riesgo pandémico en la salud, no solo para el Perú sino para el mundo, estas fiestas del Bicentenario se dan en un contexto social, económico y de incertidumbre política que tiene dividido a la población. Por esos desajustes, la arquitectura y urbanismo debe jugar un rol de liderazgo y buscar debates ante las instituciones públicas y privadas, en la academia y la sociedad civil, sobre la necesidad de implementar reformas necesarias en el campo del desarrollo urbano-arquitectónico que nos permitan enfrentar el futuro con esperanza y optimismo en el confort que nos debe brindar el hábitat (BIRF, 2020). Desde este punto de vista, la historia de la arquitectura, como disciplina, cumple un rol primordial para la reflexión y analizar con nivel crítico el pasado para de esta manera ser capaces de plantear alternativas de cambios hacia el bicentenario.

La independencia del Perú debe ser tomada como punto de inflexión entre la arquitectura moderna y la arquitectura contemporánea que se tiene en la actualidad y entendiéndola como un proceso histórico de cambio.

Uno de los principales retos del Bicentenario del Perú es alcanzar la sostenibilidad urbana a lo largo y ancho del país, lo cual plantea la necesidad de disponer a futuro de un sistema que integre las ciudades y centros urbanos, alimentados por vías de comunicación, equipamientos, que conlleven a mejorar las formas de convivencia entre los seres humanos. Las instituciones competentes deben de tomar el liderazgo que permita conducir un desarrollo planificado de la urbe, con una atención preferencial a las poblaciones desatendidas y velar por que no exista un urbanismo de la pobreza basado en el tráfico de terrenos que nada bueno trae a los ciudadanos.

Pero también al ser la arquitectura parte del mundo del arte se debe promover sus proyectos culturales que tanta falta hacen a lo largo y ancho del país. ACHAT: "Un pueblo sin cultura, es un pueblo sin alma", bajo esta premisa las ciudades deben de contar con espacios tanto cerrados como abiertos para cultivar las manifestaciones artísticas, espacios en que la juventud y la ciudadanía en su conjunto puedan hacer uso de dichos espacios.

CEPAL, (2016), mediante la Agenda 2030 y los Objetivos de Desarrollo Sostenible, plantea aspectos de suma importancia sobre: "Lograr que las ciudades y los asentamientos bumanos sean inclusivos, seguros, resilientes y sostenibles" los que se deben se considera dentro de las metas del objetivo 11,11.b lo siguiente: 11.b "De aqui a 2020, aumentar considerablemente el número de ciudades y asentamientos humanos que adoptan e implementan politicas y planes integrados para promover la inclusión, el uso eficiente de los recursos, la mitigación del cambio climático y la adaptación a él y la resiliencia ante los desastres, y desarrollar y poner en práctica, en consonancia con el Marco de Sendái para la Reducción del Riesgo de Desastres 2015-2030, la gestión integral de los riesgos de desastre a todos los niveles"; desde ya se debe considerar las medidas adecuadas por medio de la planificación urbana e ir dando cumplimiento a estas recomendaciones.

Por lo que, siendo la arquitectura un componente fundamental de la cultura de las ciudades, es una de las manifestaciones humanas más importantes, que constituye la identidad de las sociedades y permite resaltar su pasado, reconocer su presente y planificar su futuro. 


\section{ROL DEL ARQUITECTO}

La arquitectura y el urbanismo son expresiones espaciales objetivas de la conducta humana en la esfera de su asentamiento, en la conducta humana hay muchos aspectos que no cambian, la felicidad, la tristeza, el amor, la muerte; durante el transcurrir del tiempo ha existido diferente relación entre el hombre y el espacio, el espacio no tiene lugar ni el tiempo momento para el hombre. Este es excluido para poder incluirlo-ayudado a volver al hogar- el hombre debe ser integrado en sus significados. El hombre es el sujeto y al mismo tiempo el objeto de la arquitectura, pues es espacio en la imagen del hombre es el lugar, y el tiempo en la imagen del hombre es la ocasión. Por tanto, el hombre debe ser considerado dentro del espacio actual en la arquitectura de manera que se sienta confortable y confortado, en estas épocas de pandemia se le debe dotar de espacios libres, espacios verdes para poder oxigenar todo el cuerpo y no sentirse agobiado dentro de un ambiente.

Solo el pensar creativo del arquitecto posibilitara al hombre a expresar su derecho a vivir de un modo individual y personal de vida, a través del volumen construido.

Tenemos un ejemplo de cómo se genera un asentamiento humano por medio de la migración de las personas a través del tiempo y encuentran espacios urbanos sin ningún tipo de planeamiento lo que genera normalmente problemas de vulnerabilidad física y social que surge a través de procesos de ocupación informal incontenible.

Según el (INEI, 2020), en el censo 2017, se estimó que Huancayo metropolitano contaría con 608.217 habitantes para el año 2020.

\section{Gráfico 1}

Plan de Desarrollo Urbano 2006-2011
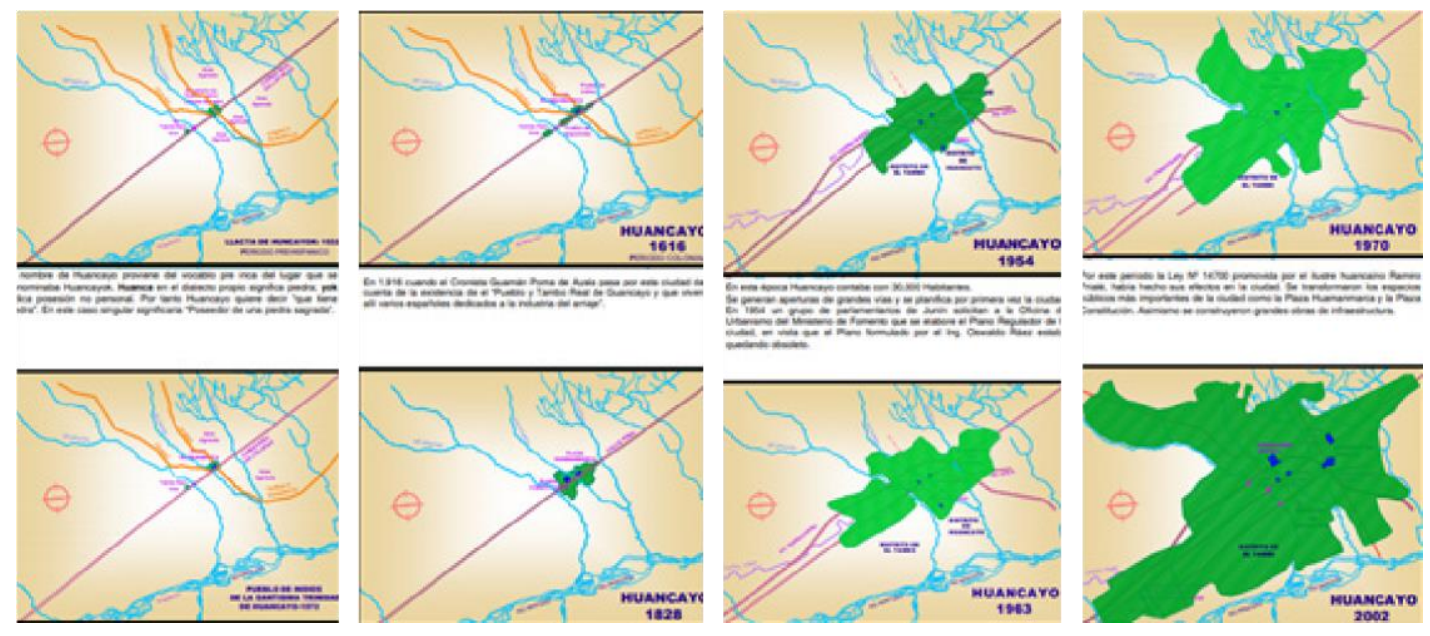

Fuente: Modificado de (Ricse Suasnabar, 2006)

La llegada vertiginosa de migrantes de las diferentes provincias hacia la ciudad de Huancayo agarro desprevenidos a nuestras autoridades quienes en los municipios respectivos no contaban con un documento de planificación elaborada el mismo que debería de regir y organizar el crecimiento armónico de la ciudad a la vez debería de incorporar a estas personas al tejido urbano social. Sin embargo, esta llegada masiva de familias, estos migrantes se asentaron en territorios disponibles, generalmente en la 
periferia de la ciudad creando problemas urbanos al no contar con los servicios básicos agua, desagüe, fluido eléctrico, pistas, veredas, entre otros-.

La mayor parte de estas "manchas urbanas" se organizaron autogestionariamente y tuvieron la capacidad para construir sus viviendas, luego convertirlos en barrios como esfuerzo principal para generar ciudad (BID, 2016). Contando intermitentemente con apoyo de las instituciones, los vecinos y vecinas conformaron comités o juntas vecinales, representadas por juntas directivas elegidas por sus integrantes, encargadas no sólo de coordinar los esfuerzos de la comunidad, sino también de ser interlocutores con las diferentes instancias públicas, las organizaciones vecinas o las estructuras organizativas sociales de mayor escala.

Las juntas vecinales jugaron un rol primordial para el desarrollo inicial de los diferentes barrios, que poco a poco se fueron consolidando con la intervención subsidiaria del Estado: Implementación de los servicios de infraestructura básica, transporte público y el sistema educativo, para los que se dejaron áreas de aporte, en previsión de los equipamientos que debieran ejecutarse posteriormente. Por otro lado, el sistema de propiedad y lotización (división de los terrenos) fueron trabajados y/o titulados por COFOPRI respondiendo a un modelo extensivo de ocupación, con lotes pequeños para el desarrollo de viviendas unifamiliares autoconstruidas, - a menudo cada piso corresponde a una nueva generación o núcleo familiar.

Esta nueva sociedad da al hombre la oportunidad de mantener una relación individual con la vida dotándoles de condiciones físicas, medios técnicos, psicológicas, espirituales y estéticas que les permiten organizarse de una mejor manera.

\section{LA CREATIVIDAD EN EL CAMBIO URBANO}

Cuando nos desplazamos por las diferentes ciudades pensando en la relación que debe existir entre la creatividad y desarrollo de los objetos arquitectónicos, sorprende al observar qué poco ha cambiado en estas últimas décadas del siglo XX las propuestas de mejora de las ciudades, o es que no entendemos aún que nuestro cerebro cuenta con una parte plástica espacial que relaciona las formas, proporcionas, colores, las diferentes variables de la arquitectura y lo más importante la triada de Vitrubio: firmitas, utilitas, venustas, elementos que nos ayudan a proponer ideas muy creativas con el fin de ordenar el espacio que habitamos. El concepto de creatividad está relacionada a las artes visuales como la escultura, pintura y arquitectura, pero existen disciplinas como la pedagogía y la psicología que han sido las primeras en estudiarla, los arquitectos estamos persuadidos de que la arquitectura por medio del acto creativo ofrezca volúmenes, espacios arquitectónicos y urbanos para el confort de los usuarios y recrear la percepción visual realizando un esfuerzo creativo conducente a la producción de soluciones originales.

A pocos meses de cumplirse el bicentenario del Perú es importante considerar y valorar tanto la originalidad, la novedad, la innovación, la flexibilidad, la fluidez en la producción de la arquitectura sin olvidarnos de proponerlos, para que nuestras ciudades ofrezcan soluciones creativas a los miles de usuarios.

La educación en la arquitectura como en el ejercicio de la profesión, asume que el ser creativo es la capacidad de producir algo novedoso por medio de los procesos 
mentales teniendo como objetivo la solución de un problema determinado, por tanto, es un concepto que debemos cultivarlo sabiendo que no es exclusividad de la arquitectura, pero juega un rol relevante en el diseño arquitectónico. Como lo menciona Einstein en la publicación de (Romo, 2018) "No tengo dotes especiales solo soy apasionadamente curioso".

Se puede asegurar que la investigación está comprometida con la creatividad debido a que son dos procesos que tienen un objetivo en común, el descubrir algo nuevo a raíz de sucesos anteriores, por tanto, investigación y creación se sustentan en el pasado con proyección al futuro.

Hoy ad portas del bicentenario se debe de poner en práctica en la misma ciudad que es el gran escenario espacial, generar investigaciones creativas conociendo que la creatividad urbana es un factor esencial que da forma al concepto y diseño de proyectos urbano arquitectónico vinculados al espacio público, al ocio, a las industrias creativas, a la economía creativa, la resiliencia y talento creativo. Es la manera en que los procesos creativos se manifiesten en la ciudad: el modo en que el ingenio y la creatividad, entendida como la capacidad humana de transformar un estado o situación en otra, impacta en las ciudades.

¿Por dónde va la arquitectura y el urbanismo hacia el bicentenario?, es lógico pensar que se tiene que encontrar nuevas ideas de desarrollo urbano e ir proponiendo la construcción de nuevas ciudades "creativas" que brinden lo que adolece en la actualidad seguridad, economía, educación, salud, espacios libres para la recreación, transporte, modelo de ciudad que brinde la habitabilidad adecuada para el ser humano del siglo XXI, las edificaciones deben contar con estructuras arquitectónicas bajas y nivel medio para no deshumanizar a la persona y que brinden bienestar a los niños y personas adultas.

\section{REFERENTES ACTUALES DE CIUDADES A CONSIDERAR}

Para Suzuki et al., (2019) Singapur, es una de las ciudades modernas que cuenta con los equipamientos más sofisticados del mundo y con unas inversiones extraorbitantes.

Singapur es una isla estado, ubicado al extremo sur de la península de Malasia.

Población: Aprox. 5,7 millones de habitantes (2019).

Idioma oficial o principal: inglés, chino (mandarín), malayo (bahasa) y tamil.

Hoy Singapur es la ciudad-estado más próspera de Asia y del mundo, reina de la eficiencia energética y símbolo de modernidad. Es una mezcla de capitalismo privado e intervencionismo estatal. Una nación líder en educación, sanidad y competitividad económica.

Los ciudadanos a menudo piensan en la posibilidad de combinar una serie de elementos aparentemente antagónicos para lograr un entorno urbano amigable y sostenible. Una ciudad con gran actividad comercial y financiera complementada con programas sociales para la vivienda. 


\section{Gráfico 2}

Imagen central de Singapur

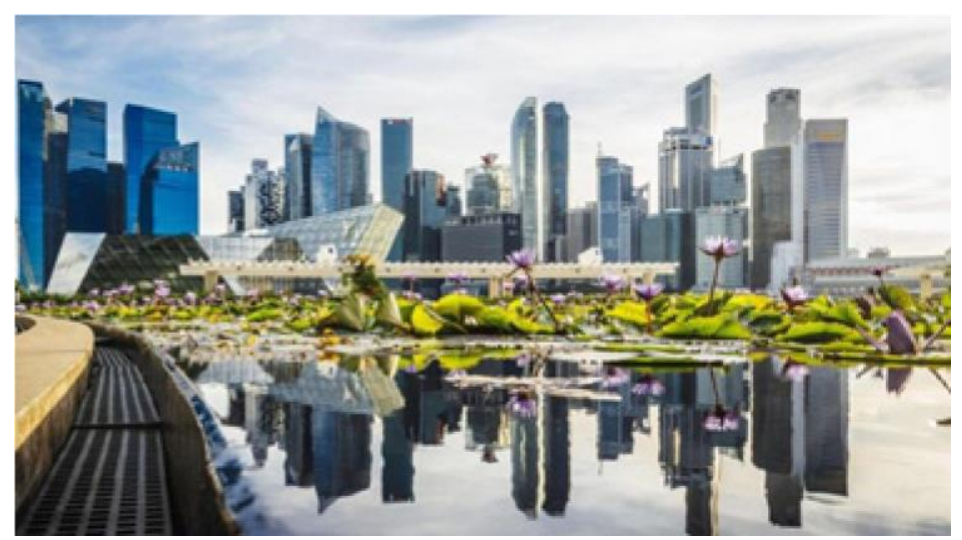

Fuente: (BBC News Mundo, 2019)

Una comunidad que compense la elevada densidad demográfica con viviendas asequibles en zonas ajardinadas y con mezcla de culturas y etnias sin que se produzcan áreas marginales y lugares excluyentes. Todo ello, con lugares públicos limpios y acogedores, gracias a la disciplina y buenas prácticas de sus habitantes.

Es importante mencionar como la arquitecta Maria Boey tuvo una participación directa en este cambio del fenómeno de sociedad urbana moderna:

Cuando le hacen una pregunta: "Singapur aparece como un fenómeno de referencia internacional por la coordinación de la actividad financiera, disciplina ciudadana y respeto al medio ambiente. ¿Podría indicar cuales son las claves del éxito?

La respuesta se cae por si sola haciéndonos conocer la idea fuerza que sirvieron para esta transformación, "Uno de los retos medioambientales ha sido tratar de poner la ciudad en un jardín, no solo jardines dentro de la urbe. Aparte de las políticas económicas y sociales, uno de los elementos clave ha sido la participación de sus habitantes en el diseño y mantenimiento de zonas verdes, en sus distintas categorías. Hay parques infantiles con juegos para niños, áreas para adultos con equipos de ejercicio físico, circuitos de paseo y ciclismo, bancos y refugios para la lluvia, combinado con paredes y azoteas verdes. Tenemos, además, grandes parques, como el Jardín Botánico, que es un referente de la UNESCO, con especial protección a ciertas especies vegetales, como orquideas..."

La sostenibilidad es una característica esencial en la naturalización urbana (movimiento que promueve el enverdecimiento de las ciudades). ¿Cómo se ha enfocado en Singapur?

"La sostenibilidad es un concepto muy amplio que va desde el diseño de edificios, materiales a utilizar y producción de alimentos. Entre los principales objetivos del Gobierno se encuentran el alojamiento para el 80\% de la población de las ciudades, el empleo, la educación, el medio ambiente y la salud. Dichos objetivos se incorporan a la planificación urbanistica y son asumidos por todas las Autoridades. La idea de la ciudad verde se inició en la Junta de Parques Nacionales para conservar la naturaleza, flora y fauna, manteniendo los recursos naturales".

Siendo una de las ciudades más conocidas del mundo, no es de sorprender que Dubai sea la ciudad ecológica del futuro, tendrá la más pequeña tasa de contaminación 
mediante la implementación de la "estrategia de energía limpia" en el año 2050 (Van Ploeg, 2021).

El tema de la sostenibilidad se ha vuelto progresivamente importante en los Emiratos Árabes Unidos en los últimos cinco años, desde que Dubai anunció su objetivo de ser una de las ciudades más sostenibles y ecológicos del mundo.

Dubai logra ser una ciudad sostenible y ecológica porque aplica características muy importantes para su cambio (Sánchez Hernández, 2015).

- Reciclar el agua

- Recolectar y reutilizar el agua de lluvia para reducir la demanda de agua potable

- Implementar sistemas de riego altamente eficientes e inteligentes valiéndose del agua pluvial

- Instalación de accesorios, medidores de agua y electrodomésticos eficientes

- Manteniendo y mejorando la calidad del agua subterránea y superficial

- Diseñando un paisajismo de bajo uso de agua

- Reduciendo el consumo de agua en un 50\% al aumentar la conciencia ambiental

\section{LA NUEVA PLANIFICACIÓN}

La arquitectura y el urbanismo con miras al Bicentenario del Perú, deben de contar con una propuesta muy clara para realizar ideas creativas y coherentes con una visión de futuro, la propuesta de la fundación de nuevos asentamientos humanos que son muy necesarios en los momentos actuales debido a que las ciudades están creciendo muy desordenadamente produciendo graves problemas de habitabilidad y congestionamientos por lo que se tiene que ordenar y resolverlas de la mejor manera, existen planes de desarrollo urbano, planes metropolitanos de desarrollo, entre otros planes de desarrollo de las ciudades los que no son aplicados en toda su magnitud, además deben de pasar a la obsolescencia debido a que fueron elaborados antes de la pandemia del Covid 19.

Nos hemos acostumbrado a no respetar los espacios intangibles como los de agricultura que son invadidos para sembrar cemento los que traerán resultados funestos en un futuro no muy lejano, los agentes inmobiliarios son indolentes con el aparato productivo y solo ven desde un aspecto económico y no social, por lo que las nuevas propuestas deben de respetar el medio ambiente y la agricultura. Las nuevas poblaciones deben de estar ubicadas en zonas eriazas o lomas de los cerros que existen muchos en nuestro país, algunos están regados por las aguas de los ríos los mismos que se pierden sin misericordia estos deben de aprovechados para construir diques o represas para evitar la falta de agua en las ciudades. Estas nuevas ciudades deben integrar estrategias productivas y logísticas bajo un arquetipo sostenible para enfrentar los problemas del cambio climático. Por otro lado, debe existir voluntad política para obtener la viabilidad política, económica, social y no se debe dar cabida a la corrupción.

No hay nada imposible, cuando se nos viene a la mente de cómo se construyó Venecia sobre el fango hace poco más de 15 siglos (Governeur, 2021b). La pandemia nos ha dejado mucha incertidumbre que ha removido todas las estructuras de la sociedad, la ciudad, como el modelo de vida imperante, parece que debe repensarse de una vez por todas. 


\section{Gráfico 3}

Venezuela, perfil principal de la ciudad

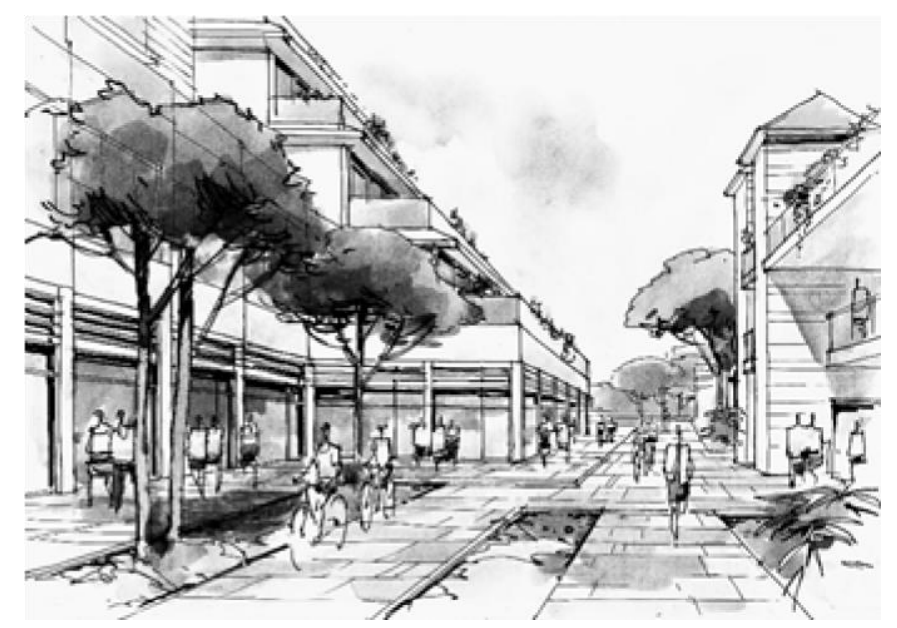

Fuente: (Quintana Urnaga, 2016)

Las ciudades atomizadas de personas, vehículos motorizados y no motorizados, sin espacios para estacionamientos, gran cantidad de vías tomadas por la informalidad de los comerciantes, vías sin veredas para la circulación de las personas, el smog, ese modelo debe ser reemplazado por un nuevo modelo urbano, por otros más saludables, incluyentes y respetando el medio ambiente para que sean sostenibles.

El arquitecto, urbanista y académico Governeur, (2021), plantea paradigmas o modelos que deben de cambiar en las ciudades, la ciudad "carrocentrista" no debe ir más, porque desplaza al peatón. La ciudad debe ser policentrica y no requerir de tantos viajes en auto, los servicios y oportunidades para las personas deben estar disponibles a solo 20 minutos como máximo. Deben ser ciudades espacial y socialmente integradas. Deben tener una densidad intermedia para evitar el hacinamiento y humanizar la misma y por último se debe hacer un estudio real del aspecto económico y productivo familiar. El objetivo debe ser realizar una "acupuntura" a la ciudad.

Sería interesante tener la economía de los países del primer mundo para hacer propuestas de diseño urbano-arquitectónico con todos los equipamientos que requiere la comodidad humana en todo el sentido de la palabra, pero hay que poner los pies sobre la tierra.

La crisis de las ciudades actuales tienen su origen dentro y fuera de ellas mismas, la sociedad sus gobiernos y el medio ambiente se están deteriorando por falta de una buena planificación más aún que siguen albergando y recibiendo a los migrantes de diferentes lugares de la región y de la nación, los mismos que provocan alteraciones en el equilibrio de las poblaciones rurales y urbanas y sus consecuencias son que debilitan las bases económicas; pero así como existen desequilibrios estas sirven como incentivo para hacer nuevas propuestas de un nuevo centro poblacional urbano para solucionar estos desequilibrios existentes.

El procedimiento debe ser eficaz para solucionar los problemas integrales y no solo hacer propuestas en el sector vivienda, sino la de protección de los espacios agrícolas 
intangibles que en la actualidad vienen siendo alterados por el crecimiento del cemento que no brinda raíces productivas más bien ayudan a crear islas de calor.

Para que realmente exista un cambio en la estructura urbana de las nuevas ciudades, de acuerdo con William Alonso, citado en (CEPAL, 2016b), se deben de proponer políticas: "Una política para la urbanización debe contener objetivos para guiar el desarrollo del fenómeno de crecimiento.......". Por política conocemos "...como una estructura claramente definida de planes generales o de principios fundamentales, seleccionados entre varias alternativas, tomando en cuenta las condiciones existentes y las previstas, para guiar o modificar las decisiones o los cursos de acción".

Las características de la política se pueden definir en tres:

a. Es una definición de metas y objetivos y que se constituyen por principios que se interrelacionan por sistemas de reglamentación coordinados para ciertos fines.

b. Proporciona un conjunto de estrategias definidas, siendo necesarios que existan alternativas antes de que se dé la forma final.

c. Se debe incluir análisis de ideas de agentes externos, definición de principios y presentación de una ruta de trabajo para una solución final coherente.

En contraposición existen problemas en la política de crecimiento urbano en cuanto a determinar su campo de acción por tanto es necesario seleccionar el lugar adecuado y las características de la sociedad resultante.

Para que una ciudad funcione orgánicamente se propone un modelo de políticas que servirán de norte para la toma de decisiones:

\section{Gráfico 4}

Planificación de nuevas ciudades



\section{Gráfico 5}

Patrones urbanos

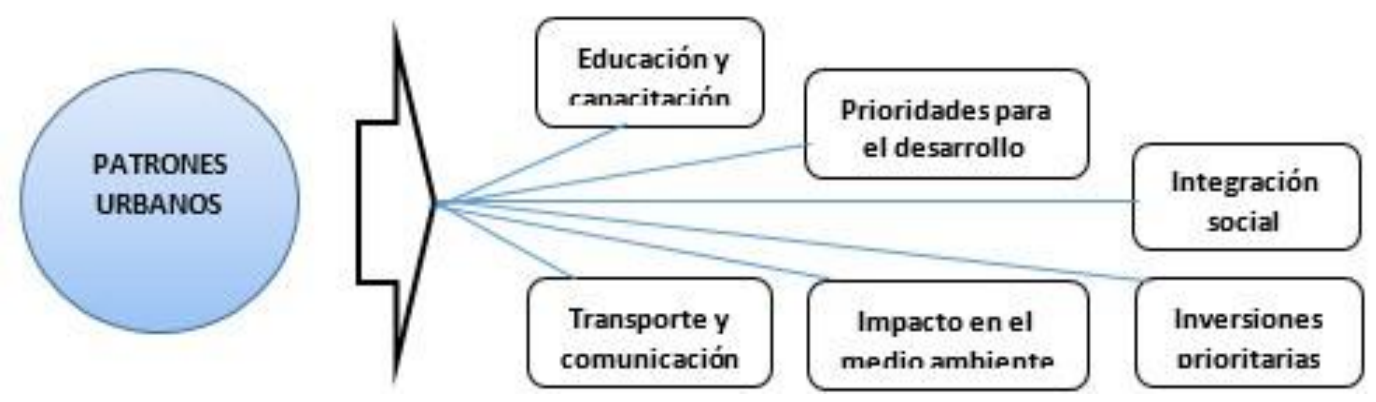

Fuente: Adaptado de (Golany, 2021) 


\section{Gráfico 6}

Formulación de politicas en la diferentes areas

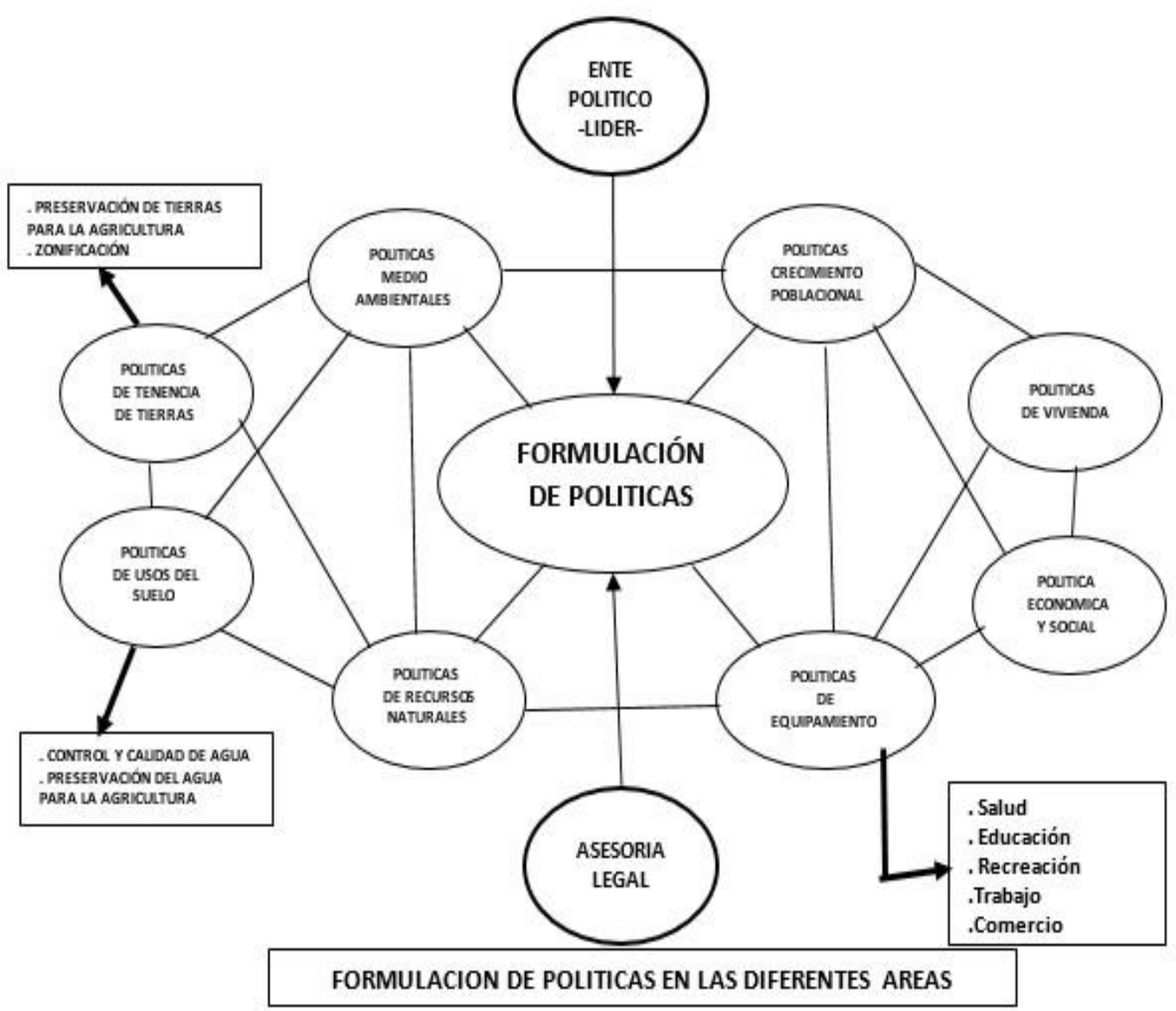

Fuente: Adaptado de (Golany, 2021)

La política de propuesta para la formación de una nueva ciudad urbana debe estar orientada a la adaptabilidad de las condiciones socioeconómicas y geográficas del lugar de asentamiento estableciendo patrones nuevos a las diferentes áreas propuestas considerando lo siguiente:

1. Localización adecuada y su interrelación geográfica.

2. Tamaño de la población en relación con el asentamiento en el aspecto físico.

3. Determinar las funciones económicas, sociales y administrativas.

Debe plantearse claramente estos tres aspectos para que la propuesta sea eficaz y pueda tener una eficiente interrelación entre los patrones urbanos.

\section{CONCLUSIONES}

- El problema de la pandemia ha sido determinante para determinar que la arquitectura y el urbanismo en las ciudades no estaban preparadas tampoco bien concebidas en toda su configuración física como ciudad para este evento.

- El elemento fundamental de la cultura es la arquitectura, la que es una de las manifestaciones humanas más importantes, que constituye la identidad de las sociedades urbanas permitiendo resaltar su pasado, reconocer su presente y planificar su futuro. 
- El crecimiento de nuestras ciudades se ha dado a un ritmo muy rápido y de una manera muy empírica sin respetar las normas y reglamentos que rigen su crecimiento ordenado.

- Sobre las características demográficas no se ha tenido ningún tipo de estadísticas que conformen una data real por ser un crecimiento espontaneo generando bolsones humanos desatendidos.

- Los retos a futuro con miras al bicentenario de nuestro país es que se disponga de un sistema integrado de ciudades y centros urbanos, alimentados siempre por formas de convivencia y de vida amparadas en una sólida educación y ética, a la vez que cuenten con los servicios básicos necesarios.

- Se debe contar con nuevos centros urbanos que se convertirán en el motor dinamizador de las ciudades del país, con proyección al mundo.

- La formación de los futuros planificadores deberá tener nuevas capacidades de la problemática de las ciudades para enfrentar el reto en el desarrollo sostenible urbano y cultural de la ciudad, para lo cual es necesario que las universidades "tengan una lectura" de las ciudades del futuro y se tome conciencia de que la formación de la juventud debe estar dirigida a la modernización de la enseñanza para que estén preparados de enfrentar los nuevos retos y obtener respuestas acordes a la actual realidad y la venidera.

- $\quad$ El Perú del Bicentenario debe ser un país ordenado, diverso y mixto. Esa diversidad supone una serie de problemas, pero a la vez constituye un gran reto para su solución creativa.

- Los peruanos del Bicentenario debemos estar orgullosos de nuestro pasado y ser conscientes de que nuestra riqueza histórica y cultural debe ser la base de un futuro promisorio.

\section{REFERENCIAS BIBLIOGRÁFICAS}

BBC News Mundo. (2019). Cómo Singapur pasó de ser una isla pobre a uno de los países más ricos del mundo (y qué amenaza enfrenta ahora). Mundo, 1. https://www.bbc. com/mundo/noticias-47032379

BID. (2016). Huancayo: hacia la sostenibilidad metropolitana bajo el liderazgo de un gobierno local moderno. Journal of Chemical Information and Modeling, 53(9), 149. https://az.b-ok.lat/book/2880647/40fc6e

BIRF. (2020). Coronavirus: La respuesta del Grupo Banco Mundial ante la emergencia mundial de hacer frente a la pandemia. Sepa más. Banco Mundial, i, 1-18. https:// datos.bancomundial.org/indicator/SP.URB.GROW?locations $=\mathrm{SG}$

CEPAL. (2016a). Agenda 2030 en América Latina y el Caribe Gracias por su interés en esta publicación de la CEPAL. https://repositorio.cepal.org/bitstream/handle/ 11362/43963/1/S1800556_es.pdf

CEPAL. (2016b). América Latina y el Caribe. Desafíos dilemas y compromisos de una agenda urbana común. ONU HABITAD, 62. https://repositorio.cepal.org/ bitstream/handle/11362/40656/1/S1600986_es.pdf 
Golany, G. (2021). PLANIFICACIÓN DE NUEVAS CIUDADES Principios y prácticas (L. Dickens (ed.); Primera Ed). https://www.iberlibro.com/primeraedicion/PLANIFICACIÓN-NUEVAS-CIUDADES-Principios-prácticas-GIDE $\mathrm{ON} / 15171344587 / \mathrm{bd}$

Governeur, D. (2021a). Así tendrán que ser las ciudades luego del COVID-19. La Network, 1-13. https://la.network/asi-tendran-que-ser-las-ciudades-luego-delcovid-19/

Governeur, D. (2021b). Las ciudades tendrán que ser MULTICENTRICAS con prioridad al \# espacio _ público. Limaparislima, 1-13. https:/ /limaparislima.wordpress.com/ 2020/07/10/la_network-las-ciudades-tendran-que-ser-multicentricas-con-priorida d-al-espacio_publico/

INEI. (2020). INEI, Base de datos. https://www.inei.gob.pe/bases-de-datos/

Quintana Urnaga, L. (2016). VENEZUELA INTERNACIONAL Habitar Dignamente la ciudad. Ministerio de Desarrollo Urbano. https://uploads.habitat3.org/hb3/ venezuela.pdf

Ricse Suasnabar, O. (2006). Plan de desarrollo urbano 2006-2011. Municipalidad Provincial de Huancayo. http://bvpad.indeci.gob.pe/doc/estudios_CS/ Region_Junin/huancayo/huancayo_PDU.pdf

Romo, M. (2018). Does creativity have a gender? Obstacles to excellence in women. Estudos de Psicologia (Campinas), 35(3), 247-258. https://doi.org/10.1590/ 198202752018000300003

Sánchez Hernández, S. (2015). El proceso de transformación urbana de dubái (pp. 1-71). https:// core.ac.uk/download/pdf/41823192.pdf

Suzuki, H., Dastur, A., Moffatt, S., Yabuki, N., \& Maruyama, H. (2019). Las ciudades ecológicas como ciudades económicas. In Eco2 Cities. Ecological Cities as Economic Cities (Primera Ed). http://documents1.worldbank.org/curated/en/ 791721468024588359/pdf/544320PUB0SPAN0064920January0502015.pdf

Van Ploeg, P. (2021). Dubai: la ciudad ecológica del futuro. Producción Sustentable, 1-5. https://www.produccionsustentable.com/index.php/2020/09/24/dubai-la-ciuda d-ecologica-del-futuro/

Velaochaga-Sacio, R. (2020). Lima, rumbo al bicentenario. En Líneas Generales, 204-216. https://doi.org/10.26439/en.lineas.generales2018.n002.2680 